\title{
Stimulated Raman scattering microscopy for rapid brain tumor histology
}

\author{
Yifan Yang*, Lingchao Chen ${ }^{\dagger}$ and Minbiao $\mathrm{Ji}^{*}, *$ \\ *State Key Laboratory of Surface Physics and Department of Physics \\ Key Laboratory of Micro and Nano Photonic Structures (Ministry of Education) \\ Collaborative Innovation Center of Genetics and Development \\ Fudan University, Shanghai 200433, P. R. China \\ ${ }^{\dagger}$ Department of Neurosurgery, Huashan Hospital \\ Fudan University, Shanghai 200040, P. R. China \\ *minbiaoj@fudan.edu.cn
}

Received 24 June 2017

Accepted 8 August 2017

Published 23 August 2017

\begin{abstract}
Rapid histology of brain tissues with sufficient diagnostic information has the great potential to aid neurosurgeons during operations. Stimulated Raman Scattering (SRS) microscopy is an emerging label-free imaging technique, with the intrinsic chemical resolutions to delineate brain tumors from normal tissues without the need of time-consuming tissue processing. Growing number of studies have shown SRS as a "virtual histology" tool for rapid diagnosis of various types of brain tumors. In this review, we focus on the basic principles and current developments of SRS microscopy, as well as its applications for brain tumor imaging.
\end{abstract}

Keywords: Brain tumor; coherent Raman scattering; label-free imaging; CARS; SRS.

\section{Introduction}

Although brain tumors only account for approximately $3 \%$ among all cancer types, they stand an important place in cancer research due to the fact that tumors of the central nervous system are second prevalent in children and adolescents only inferior to leukemia. ${ }^{1}$ Among the brain tumors, gliomas carry one of the most dismal prognoses, with a two-year survival of about $2 \%$ for patients
$>65$ and $30 \%$ for those $<45$ years. Up to date, surgery is still the most efficient treatment for brain tumors. An optimal surgical management is associated with prolonging survival time and improving neurologic functions. ${ }^{2}$

However, there remain several challenges in brain tumor resections. First, neoplastic tissues are often indistinguishable from healthy tissues during surgeries. Bright-field neurosurgical microscopes are

\$Corresponding author.

This is an Open Access article published by World Scientific Publishing Company. It is distributed under the terms of the Creative Commons Attribution 4.0 (CC-BY) License. Further distribution of this work is permitted, provided the original work is properly cited. 
hard to visualize brain tumor margins and invasive cancer cells. Hence, tumor is often left behind and more than $85 \%$ of glioblastoma (GBM) recurrences occur at the resection margins, resulting in treatment failure and poor outcome. ${ }^{3,4}$ Conversely, unnecessary removal of normal brain tissues that do not contain cancer cells would generate neurological deficits. ${ }^{5,6}$ Second, a precise assignment of the tumor type and grade is significant because first-rank medical treatment varies widely depending on histological subtypes. Although some tumors of the central nervous system have their own individual forms of gross appearance, most of them are indistinguishable from each other. ${ }^{7}$ The importance of intraoperative histological classification during tumor surgery has been recognized for nearly 85 years. $^{3}$ Third, diverse neuropathologies such as demyelination, primary CNS lymphoma or radiation injury caused by treatment of radiotherapy to known tumors are difficult to be distinguished from glioma by magnetic resonance imaging (MRI) or computed tomography (CT).$^{8,9}$ Thus, the risks of surgical resection are high for patients with aforementioned non-neoplastic conditions.

Hematoxylin and eosin (H\&E) staining has been used as the gold standard histopathology method to diagnose brain tumors. ${ }^{5}$ Eosin stains proteins and cytoplasm bright pink, while hematoxylin stains basophilic structures (such as DNAs) blue-purple. However, $\mathrm{H} \& \mathrm{E}$ is a slow process that requires biopsy, fixation or freezing, thin sectioning and staining, thus cannot serve as an intraoperative tool. Even the intraoperative frozen sectioning, staining and histopathological consultation are still time- and labor-intensive ( $\sim 30 \mathrm{~min})$ and may delay clinical decision-making during surgery. Therefore, imaging methods delivering rapid and accurate diagnosis are highly desired for brain tumor resections.

\section{Current Technologies}

Advances have been made using a range of techniques to detect brain tumors to complement traditional histology. Noninvasive approaches for brain imaging to be stated here are CT, MRI, and positron emission tomography (PET), ${ }^{10}$ while they are largely limited by lower spatial resolution and intraoperative compatibility. Preoperative MRI coregistered to patients has been used as a navigational guide to surgeries. However, it is unable to detect the full extent of cancer cell invasion and differentiate between radiation injury and recurrent malignancy. ${ }^{11}$ The shift of brain tissues during surgeries further reduces the spatial accuracy. ${ }^{12}$ Intraoperative MRI has shown great potential in providing updated images during surgeries, but is limited by its high cost and prolonged surgical duration. ${ }^{13}$ Fluoro-ethyl-tyrosine positron emission tomography (FET-PET) has been developed and tested as a tool to guide the resection of grade 3 to 4 gliomas with $88 \%$ sensitivity, but with only $54 \%$ sensitivity for lower-grade gliomas. ${ }^{14}$ Ultrasound (US) and optical coherence tomography (OCT) have been shown to provide structural information in real time. However, US is only accessible to large scale and difficult to detect microscopic invasion, ${ }^{15}$ and OCT lacks molecular specificity. ${ }^{16}$

A range of optical microscopy technologies has been developed and applied for intraoperative imaging. Fluorescence imaging of GBM involves labeling with fluorescing molecules such as fluorescein, protoporphyrin IX or 5-aminolevulinic acid (5-ALA) which is promising to reveal brain tumor margins, yet suffers from several restrictions. ${ }^{2,17,18}$ First, only $\sim 80 \%$ of the cancer cells absorbed the fluorescing molecules. ${ }^{2}$ Second, the nonspecific labeling of dyes does not allow for tumor typing. Third, fluorophores may tend to photobleach under laser irradiations. Confocal microscopes have been applied for intraoperative imaging of tissues with fluorescence labeling, yet bare similar limitations of fluorescence imaging. ${ }^{17}$ Nonlinear optical imaging techniques have also been applied in imaging brain tissues. Second harmonic generation (SHG) microscopy is only specific to noncentral symmetric contents such as collagen fibers and microtubules $^{19}$ and third harmonic generation (THG) microscopy is sensitive to refractive index inhomogeneity, but could not provide enough molecular information. ${ }^{20}$

The emerging platform for in vivo vibrational spectroscopic imaging delivers a new way to address these problems in current neuropathology and neurosurgery. Fingerprint vibrational spectra of molecules can be recorded by infrared (IR) or Raman spectroscopy. ${ }^{21-23}$ Fourier transform infrared (FTIR) is able to perform chemical imaging of malignant gliomas and identifying brain tumor metastases. ${ }^{24,25}$ However, intraoperative IR microscopy is hampered by the strong water 
absorption and low spatial resolution given the longer wavelength $(\sim 2.5-10 \mu \mathrm{m}) .{ }^{26}$ Raman spectroscopy of brain tumors have been widely applied in rodent models and ex vivo human brain tissues. ${ }^{27,28}$ However, spontaneous Raman imaging of biological tissues has been limited by the weak signal intensities and slow imaging speed. Although handheld Raman probe demonstrated the capability in distinguishing tumor-infiltrated from normal brain, ${ }^{29}$ it only provides spectroscopic information that relies heavily on spectral and statistical analysis, and introduces more complexity for clinical diagnosis. Surface enhanced Raman scattering (SERS)-based methods have the advantages of significantly enhanced Raman signals during in vivo imaging, but they require exogenous labeling with nanoparticles. ${ }^{30}$

\section{Basic Principles of SRS Microscopy}

These limitations can be largely overcome by using coherent Raman scattering (CRS) microscopy, including coherent anti-Stokes Raman scattering (CARS) microscopy and stimulated Raman scattering (SRS) microscopy. In CRS, two excitation laser pulses, denoted as pump $(\omega p)$ and Stokes $(\omega s)$ interact with samples. Their difference (beat) frequency $\Omega=\omega p-\omega s$ matches the Raman-active molecular vibrations, thus coherently drives the corresponding chemical bonds and results in efficient signal generation that allows up to video-rate rapid imaging. ${ }^{31,32}$ In CARS, the anti-Stokes photons are generated at $\omega a s=2 \omega p-\omega s$ via a wave-mixing process [Fig. 1(a)]. ${ }^{33}$ Although CARS experiences resonance amplification with Raman modes, the signal persists even when $\omega p-\omega s$ is detuned away from resonance. This so-called "nonresonant background" is originated from the electronic responses of materials and adds to the real part of the third-order optical susceptibility, which distorts CARS spectra from Raman spectra and has troubled researchers for around a decade. ${ }^{34,35}$

SRS is analogous to stimulated emission process [Fig. 1(a)] and was reported back in 1962. ${ }^{36} \mathrm{~A}$ few groups made early efforts to integrate SRS spectroscopy with microscopy. ${ }^{37,38}$ In 2008, Freudiger et al. developed SRS microscopy with high frequency modulation and lock-in detection, and since then SRS microscope has become a rapidly

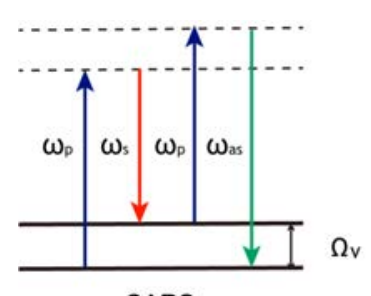

CARS

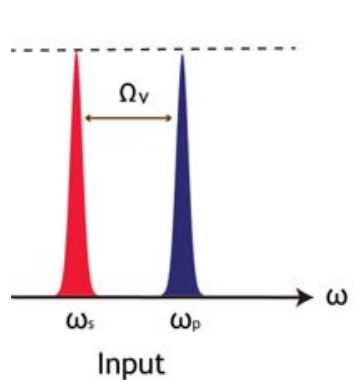

(b)

(a)

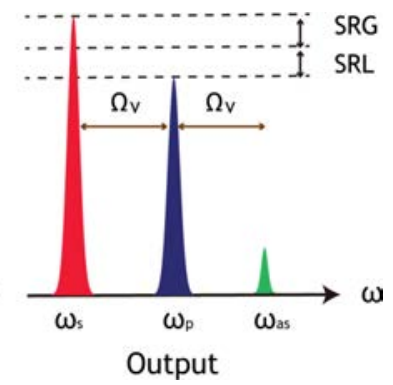

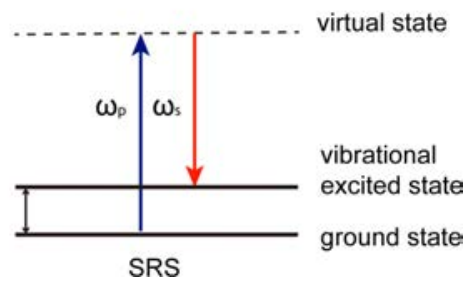

Fig. 1. Principles of CRS: (a) Energy diagrams of CARS and SRS, and (b) intensity and frequency changes in CARS and SRS processes.

growing tool for label-free chemical imaging in biomedical researches. ${ }^{39}$ SRS is known to have several advantages over CARS. First, the issue of nonresonant background was solved by SRS through self-heterodyne detection, probing only the imaginary part of the nonlinear susceptibility. SRS process results in the gain of Stokes photons (stimulated Raman gain, SRG) and a simultaneous loss of pump photons (stimulated Raman loss, SRL), and the net energy difference is converted to the vibrational energy in the excited states. Therefore, SRS could only occur at vibrational resonances [Fig. 1(b)]. Second, SRS spectra closely resemble those of spontaneous Raman scatterings without any distortion. Third, SRS signal is linearly proportional to chemical concentration, allowing easier quantitative analysis of complex system with different chemical components. ${ }^{40}$ Hence, multicolor SRS could be applied to quantitatively analyze multichemical components (such as lipids and proteins), and provide virtual histopathology of biological tissues similar to $\mathrm{H} \& \mathrm{E}$ staining. In particular, two-color SRS has been utilized to reveal diagnostic features in brain tumors, both ex vivo and in vivo, and for both animal models and human specimens. ${ }^{41-43}$ 


\section{Instrumentations of SRS Microscopy}

The basic optical setups of SRS microscopes are shown in Fig. 2. ${ }^{42,43}$ The tunable pump beam is directed from the output of an optical parametric oscillator (OPO) and the Stokes beam is the fundamental beam of the laser (e.g., $1064 \mathrm{~nm}$ ). Both beams are overlapped spatially and temporally, and interact with the samples through a laser scanning microscope. In most cases, the Stokes beam is modulated at radio frequencies $(1-20 \mathrm{MHz})$ where the noise spectrum of the laser has reached a shotnoise floor. After the sample, the pump beam is filtered out and sent to the photodiode (PD), and the SRL signal is demodulated by a lock-in amplifier. SRS imaging can be performed in both transmission [Fig. 2(a)] and reflection (epi) modes. The transmission mode is good at imaging thin tissue sections and cultured cells, whereas the epi-mode has the advantage of imaging thick tissues and live animals. ${ }^{32}$ A specially designed annular detector was used to maximize photon collection efficiency in the epi-mode, so that SRS imaging on live animals could be performed with video rate, ${ }^{32}$ and in vivo brain tumor detection could be realized. ${ }^{42}$
Multiple choices of laser sources have been used for SRS microscopy. Most common lab-based SRS microscopes are equipped with narrowband picosecond laser sources, since they readily provide high spectral resolution $\left(4-8 \mathrm{~cm}^{-1}\right)$ and sensitivity. ${ }^{44,45}$ Broadband femtosecond OPO could also be used with either spectral slicing or spectral focusing techniques. ${ }^{41,46-48}$ Furthermore, synchronized picosecond and femtosecond sources could be applied to realize hyperspectral SRS with parallel detection. ${ }^{49,50}$ Other types of multicolor and hyperspectral SRS could be found in previous works. ${ }^{51}$

Two-color SRS has been proven useful in brain tumor imaging with high sensitivity and specificity. ${ }^{42,43}$ Selective imaging at the $\mathrm{CH}$ stretching modes of $2845 \mathrm{~cm}^{-1}$ and $2930 \mathrm{~cm}^{-1}$ allows the differentiation of lipids and proteins [Fig. 2(b)]. Numerical decomposition algorithms could be applied to generate two-color maps of their distributions, forming histological images of biological tissues. ${ }^{42,44}$ However, current lab-based setups are still limited for clinical applications. Two recent technical advances have pushed SRS forward in the direction of clinical translation. First, fiber lasers (a)

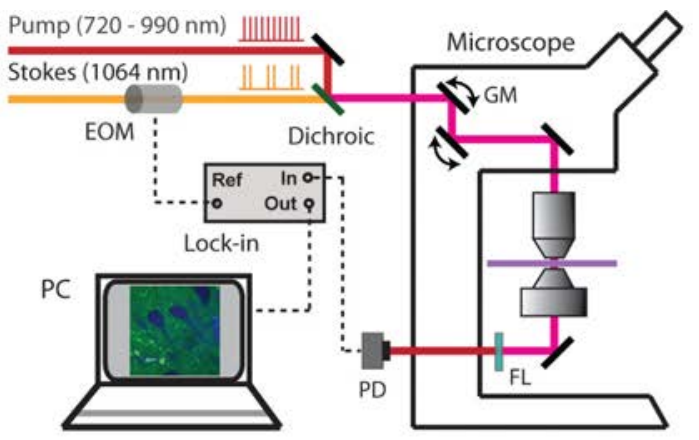

(b)

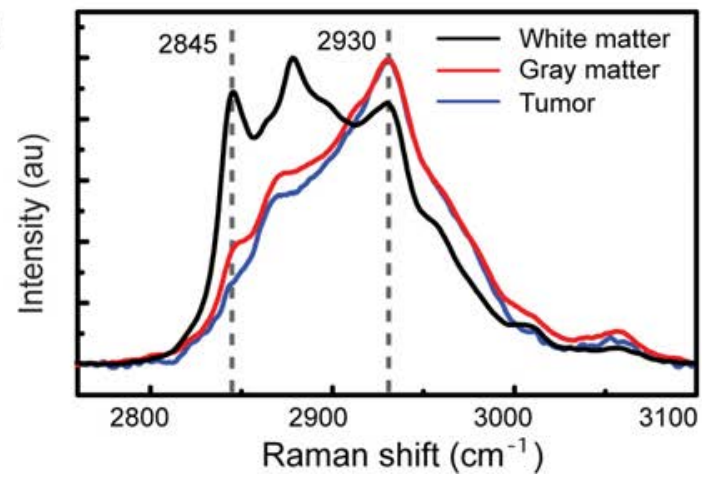

(c)

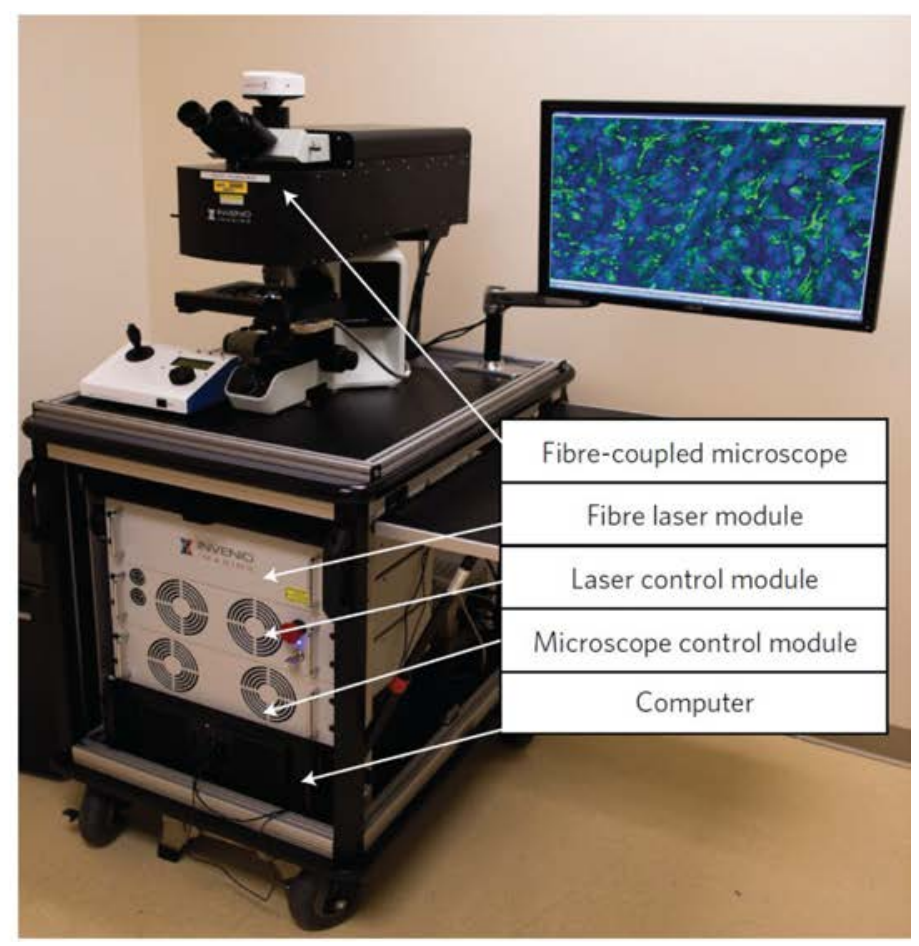

Fig. 2. Apparatus of SRS microscope: (a) Optical layouts of a typical SRS microscope, (b) Raman spectra of brain tissues in the regions of white matter, gray matter and tumor and (c) fiber laser-based SRS microscope that could be used in the operating room. EOM: electro-optical modulator, DM: dichroic mirror, PD: photodiode, GM: galvo mirrors, FL: optical filter. 
with balanced detectors were developed for high quality SRS imaging, ${ }^{52}$ thus bedside two-color SRS microscopes are available for rapid histology in the operating room [Fig. 2(c)]. ${ }^{41}$ Second, simultaneous two-color SRS imaging method has been developed with dual-phase lock-in detection, allowing real-time virtual histology without the need of post processing. ${ }^{57}$ Such a dual-phase modulation and detection method has also been applied for other imaging modalities such as transient absorption microscopy. ${ }^{53,54}$ Further developments will include the miniaturization of the microscopes into handheld devices and endoscopes for in vivo applications.

\section{Applications in Brain Tumor Detection}

CRS microscopes have been applied for brain imaging and many progresses have been made to improve the techniques and image qualities. Evans et al. conducted early trials with single color CARS microscopy detecting lipid contents, thus the tumor region showed significantly decreased signal strength due to the much lower lipid concentrations compared with normal brain tissues. ${ }^{55}$ However, such a lipid-only imaging method lacks sufficient information for accurate diagnosis. Ji et al. revealed the differences between the Raman signatures of normal and tumorous brain tissues [Fig. 2(b)], and applied two-color SRS imaging based on the contents of lipids and proteins to provide key histological information, including cellular density and morphology, axon morphology, and lipid/protein ratios. $^{42,43}$

\subsection{SRS imaging of human GBM xenograft models}

Human GBM xenografts have been used for early SRS studies to prove the working principles and perform in vivo experiments. The diagnosis of high-grade gliomas relies on the presence of specific histologic features, including significant nuclear atypia, mitotic activity, necrosis and microvascular proliferation. Frozen coronal brain sections of normal mouse brain and human GBM xenografts were imaged with both SRS and H\&E. SRS microscopy demonstrated its capability of differentiating tumor infiltration with similar efficacy to $\mathrm{H} \& \mathrm{E}$ staining [Figs. 3(a) and 3(b)]. ${ }^{42}$ While frozen sections suffer from freezing and sectioning artifacts, fresh tissue sections could provide more intact tissue (a)

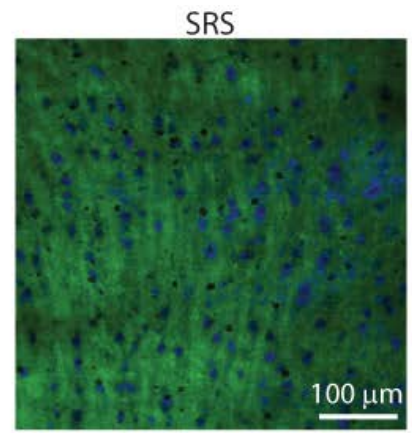

(b)

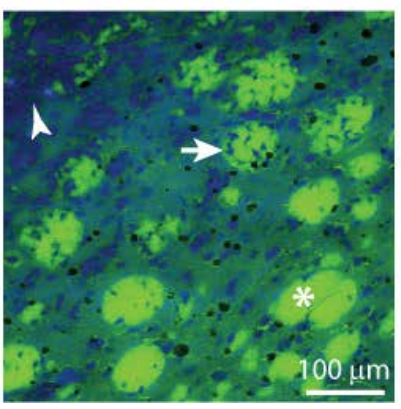

$H \& E$
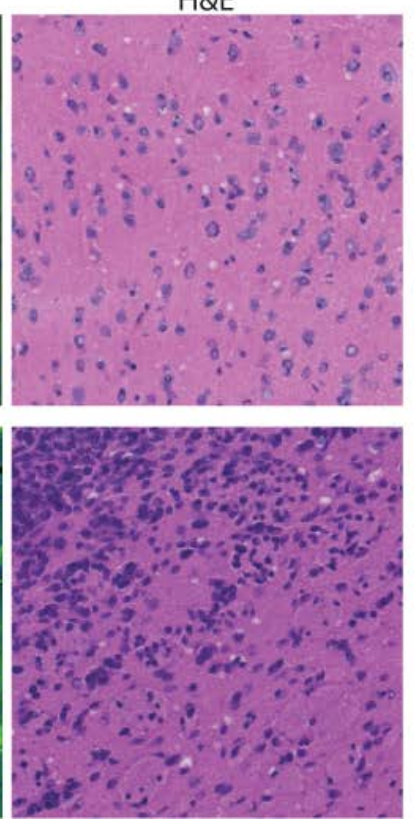

Bright field

(c)

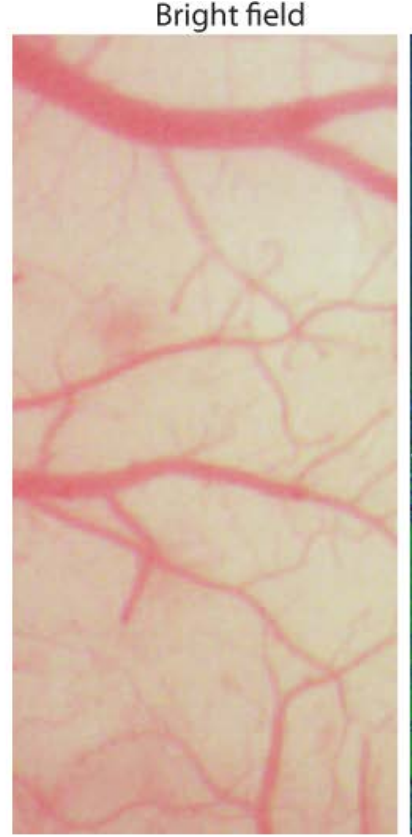

SRS

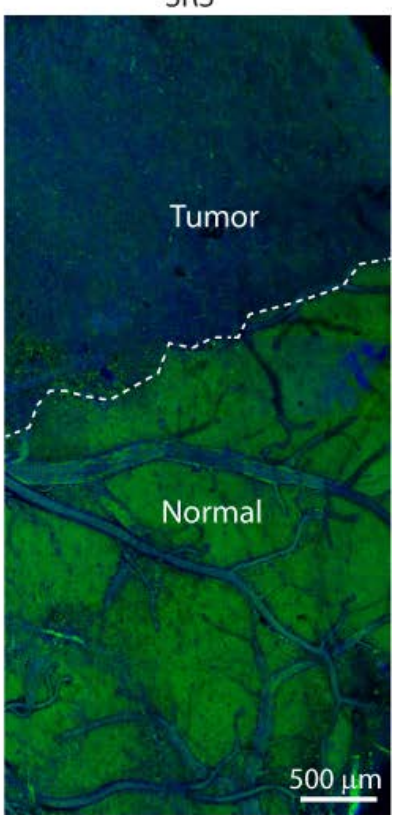

Fig. 3. Two-color SRS images of human GBM xenograft mouse brains: (a) SRS and H\&E images of a normal brain frozen section, (b) SRS and H\&E images of a GBM infiltrated brain frozen section and (c) in the field of view where bright field microscope appears grossly normal, SRS image shows a distinct margin between tumor and normal brains. 
architectures and reveal clear differences between normal and tumorous regions of a GBM xenograft mouse brain. Furthermore, since SRS is a label-free method, it has the unique capability of in vivo imaging on live animals. An infiltrative human GBM xenograft mouse model was used to recapitulate the margin of human gliomas in the cortical surface and imaged through a cranial window with epi-SRS. The regions of brain tissues that appeared grossly normal under bright-field microscope demonstrated extensive tumor infiltration with clear margins under SRS microscope based on both the chemical differences and histoarchitectures [Fig. 3(c)].

To assess the concordance between SRS and $\mathrm{H} \& \mathrm{E}$ images, web-based surveys were used to present large amount of SRS and $\mathrm{H} \& \mathrm{E}$ images in a random order to several certified neuropathologists, who were asked to classify these images into categories just as in the conventional practice. ${ }^{41,43}$ The survey demonstrates a remarkable concordance (Cohen's kappa) between SRS and H\&E, proving SRS as an effective diagnostic tool for brain tumor. This method has been used in studies of both animal models and human surgical specimens.

\subsection{SRS imaging of human brain surgical specimens}

To evaluate the ability of SRS microscopy in detecting human brain tumors, surgical specimens were used to perform quantitative ex vivo studies. ${ }^{43}$ Normal histoarchitectures of human brain tissues were obtained from different epilepsy patients, demonstrating SRS images of the gray and white matter junction [Fig. 4(a)]. As in xenograft models, while the white matters of human brain tissues contain large amount of lipid-rich myelin sheath, the gray matters possess less lipids and appear enhanced contrast of protein-rich cell nucleus. Frozen sections containing the gray-white junctions were also used to verify the origin of image contrast with combined SRS, H\&E and Luxol fast blue (LFB) staining [Fig. 4(b)].

The hallmarks of GBM in human brain tissues were studied with paired SRS and $\mathrm{H} \& \mathrm{E}$. As in the mice model, these diagnostic information include hypercellularity, nuclear atypia of viable tumor, and mitotic features (Fig. 5). ${ }^{43} \mathrm{Lu}$ et al. further reported that SRS could be used to discriminate necrosis from viable tumor [Fig. 6(a)], evaluate vascular proliferation [Fig. 6(b)], and reveal massive collagen deposition [Fig. 6(c)]. ${ }^{56}$ SRS offers major advantages over other clinically available strategies conceived to detect tumor margins as it could simultaneously image proteins, lipids, blood and components of acellular regions by tracking only a few Raman frequencies.

Retrospective and prospective researches based on postoperative imaging have shown that infiltrating glioma cells adjacent to tumor core directly give rise to recurrence in approximately $90 \%$ of cases and affect progression-free survival. A complete resection based on intraoperative detection of infiltrating gliomas by MRI and US systems is currently impossible due to the limited spatial resolution. In contrast, SRS microscopy could reveal a gradual decrease in cellularity at the margins of both high-grade and low-grade gliomas [Fig. $7(\mathrm{a})]^{43}$

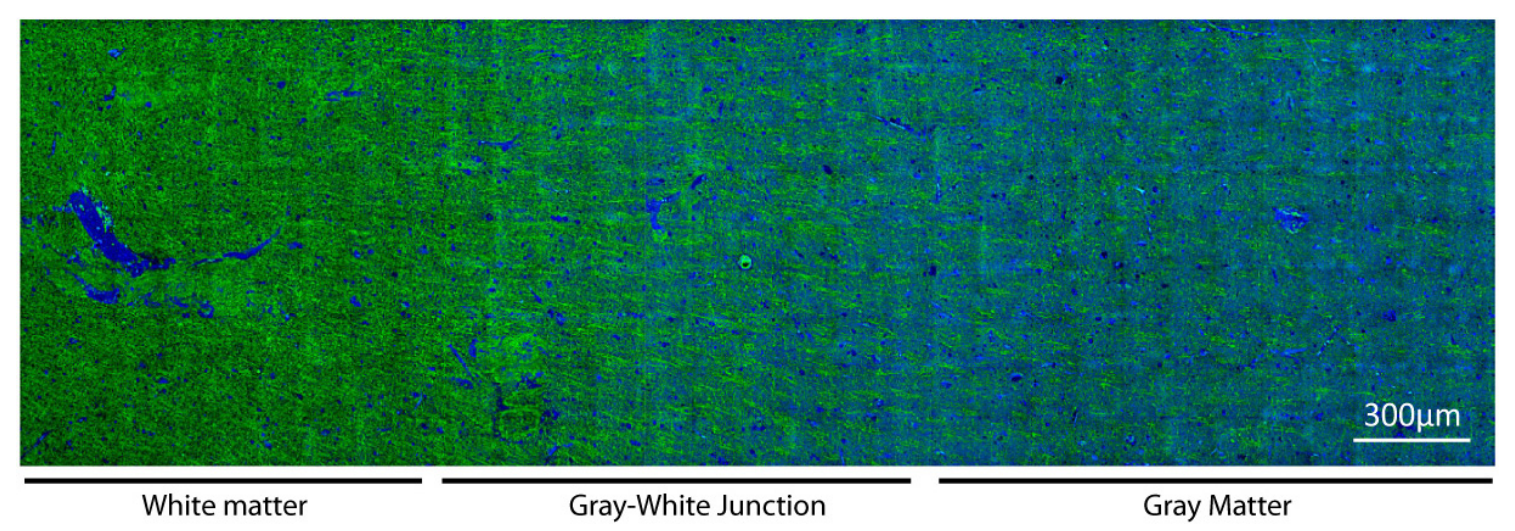

(a)

Fig. 4. SRS images of normal human brain surgical tissues: (a) Fresh tissue with a transition from white to gray matter and (b) gray-white junction imaged with frozen tissue sections, compared with H\&E and LFB staining. 


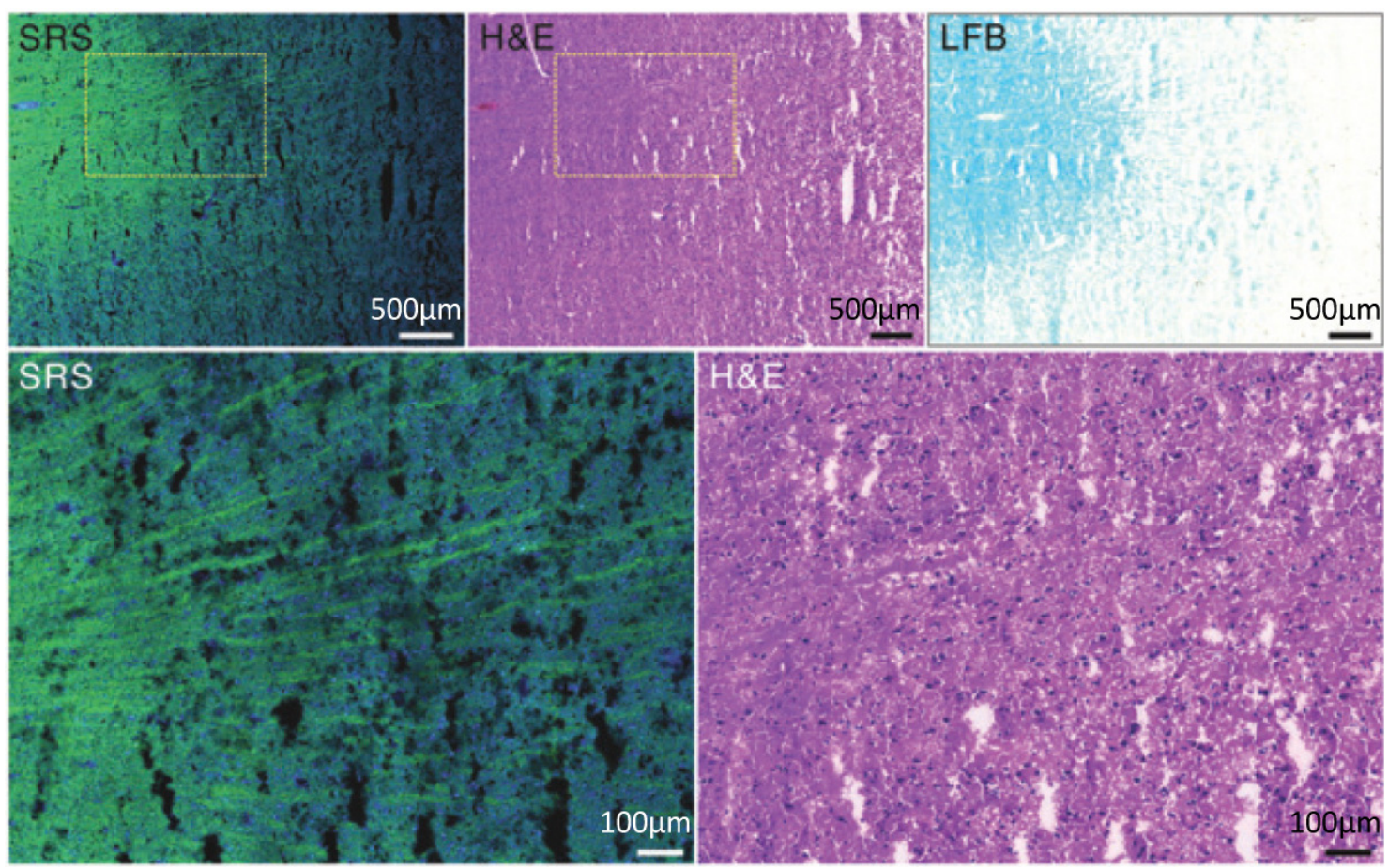

(b)

Fig. 4. (Continued)

The presence of infiltrating glioma cells in SRS images was validated by $\mathrm{H} \& \mathrm{E}$ staining [Fig. 7(b)]. ${ }^{56}$

Accurate intraoperative brain-tumor subtype diagnosis would be essential to assist in surgical decision-making during brain-tumor surgery. By devising a method that leverages SRS images to create virtual $\mathrm{H} \& \mathrm{E}$ stained slides, Orringer et al. developed the stimulated Raman histology (SRH)

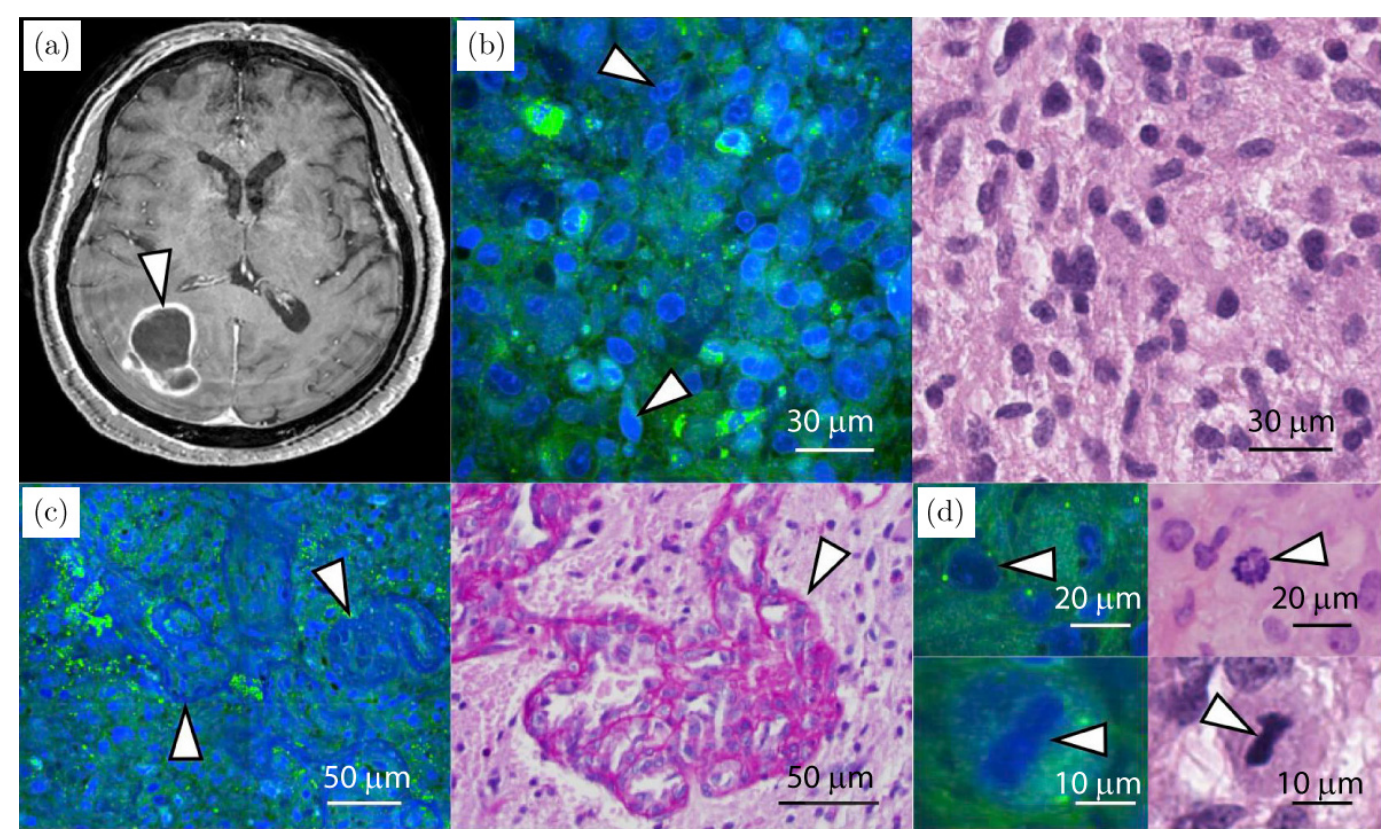

Fig. 5. SRS images of human GBM tissues: (a) MRI with enhanced ring showing GBM tissues (arrow). SRS and H\&E images of GBM tissues with diagnostic information of (b) hypercellularity and nuclear atypia, (c) abnormal microvascular proliferation, and (d) mitotic features. 


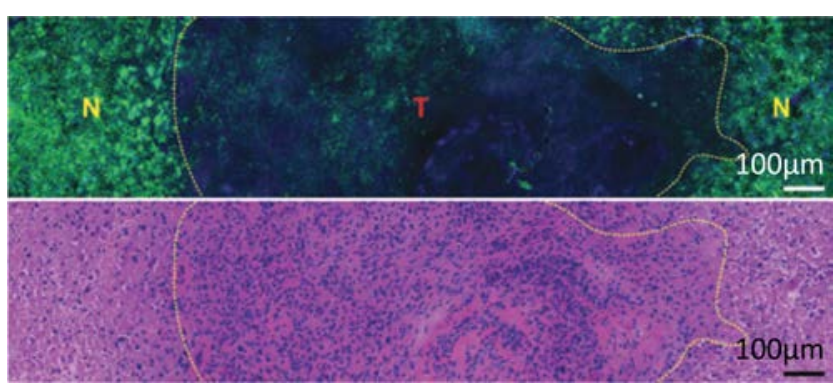

(a)

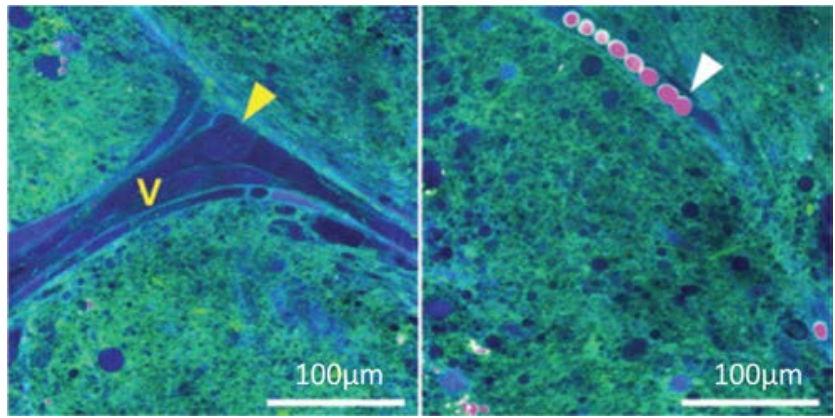

(b)

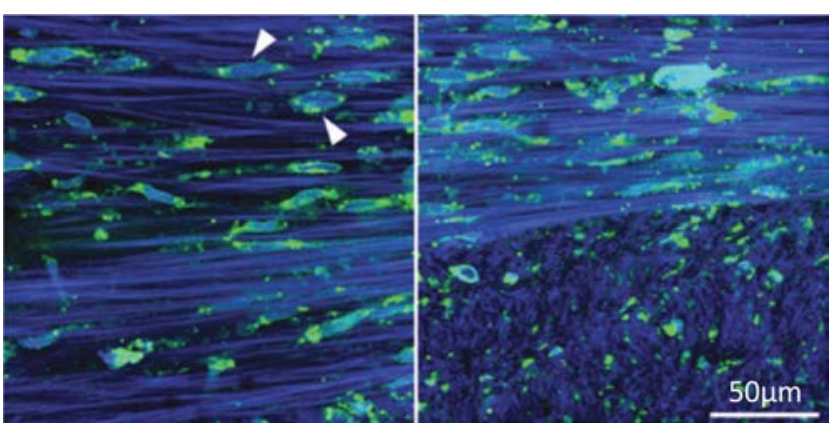

(c)

Fig. 6. Additional diagnostic feature of SRS images of human brain tissues: (a) Necrosis $(N)$ surrounding viable tumors $(T)$, (b) blood vessels $(v)$ and red blood cells, and (c) massive collagen deposition in a recurrent GBM case (gliosarcoma).

method to false color the two-color SRS images with a similar lookup table to $\mathrm{H} \& \mathrm{E}$, so that $\mathrm{SRH}$ is more familiar to surgeons [Figs. 8(a) and $8(\mathrm{~b})] .{ }^{41}$ This could be realized because $\mathrm{H} \& \mathrm{E}$ is a quasi-two-color imaging method with hematoxylin (blue) and eosin (pink) labels. Although SRS and H\&E differ in the detection of biomolecules, they could both provide key diagnostic features such as the morphologies of cell nucleus. Like conventional H\&E images, SRS reveals non-neoplastic reactive changes of normal

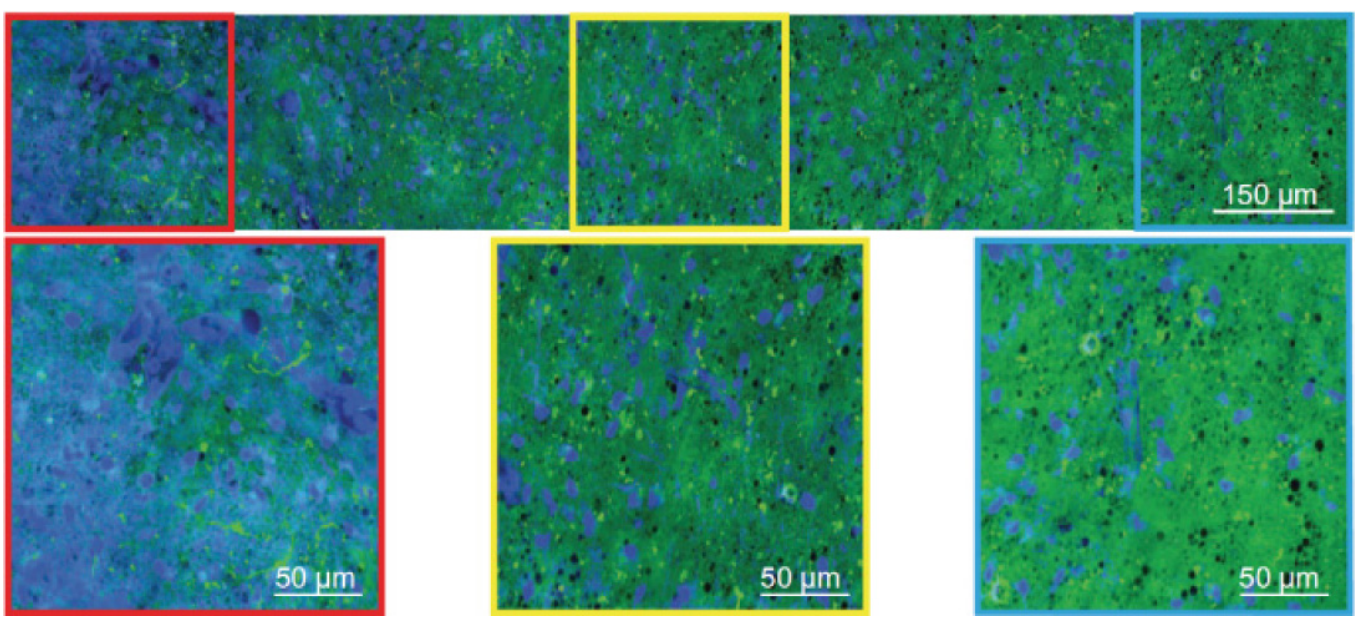

(a)

Fig. 7. SRS images showing brain tumor infiltrations: (a) Fresh human GBM brain tissue, and (b) frozen oligodendroglioma sections imaged with SRS and H\&E. 


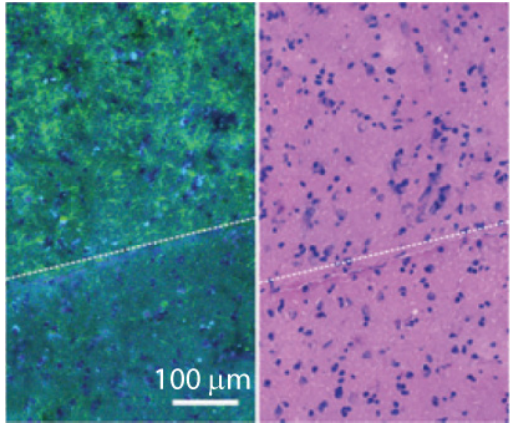

$(\mathrm{b})$
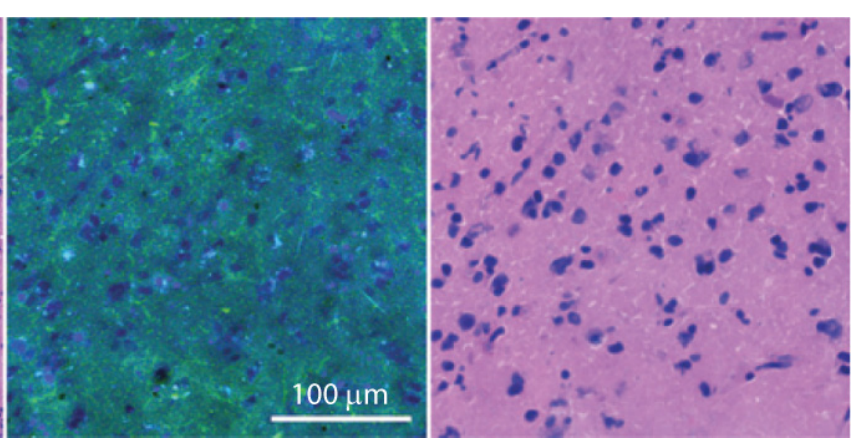

Fig. 7. (Continued)

brain tissues including gliosis and macrophage infiltration. ${ }^{41}$ SRS is also well-suited for highlighting key differences in cellularity, microvascular proliferation and nuclear architecture that distinguish
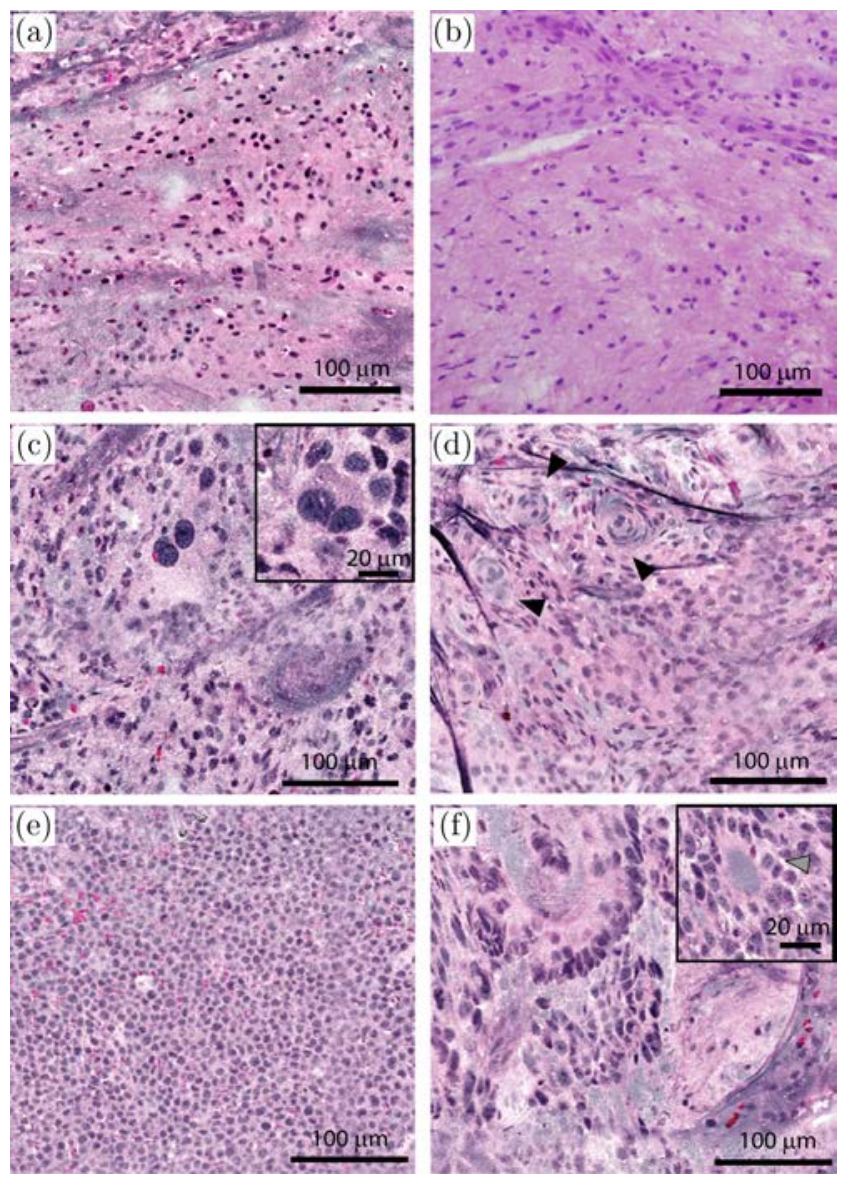

Fig. 8. SRH of human brain tumors with various subtypes: (a) A typical SRH of brain tumor with an H\&E-like lookup table, (b) H\&E image of the same tissue type as (a). Representative SRH images of (c) high-grade glioma, (d) meningioma, (e) lymphoma and (f) metastasis. low-grade from high-grade gliomas [Fig. 8(c)]. Moreover, SRS is capable of visualizing morphologic features that enable diagnosis of tumors of nonglial origin, such as meningioma, lymphoma and metastasis [Figs. 8(d)-8(f)]. SRS has also shown promises in revealing intratumor heterogeneities, which is challenging in the diagnosis and treatment of gliomas, particularly in low-grade gliomas. ${ }^{41}$

\subsection{Integration with machine learning for decision making}

Although SRS microscopy might provide an accurate, powerful and rapidly obtained interpretation of histopathologic images, it still requires the expertise of pathologists, thus remains time-intensive and prone to inter-observer variability. Automated means that ensure rapid delivering robust and consistent diagnoses would greatly incorporate SRS microscopy into the existing brain-tumor surgery workflow. A classifying program was developed based on the combination of cellular density, axonal density and protein/lipid ratio to rate the possibility of tumor occurrence within a FOV. ${ }^{43}$ Orringer et al. has recently employed a machine-learning algorithm for a more robust computational processing with multilayer perception neural network. ${ }^{41}$ The trained classifier could predict the probabilities that a given SRS image belongs to one of the four critical diagnostic classes: nonlesional, low-grade glial, highgrade glial or nonglial tumor. Machine-learningbased tissue diagnosis is expected to greatly benefit decision-making during operation, and will revolutionize intraoperative diagnosis combined with novel imaging methods. 


\section{Summary}

In summary, we have reviewed recent progresses in developing SRS microscopy as a label-free digital histology tool for imaging brain tissues. Two-color SRS detection of lipids and proteins has demonstrated success in differentiating tumor from normal brain tissues, based on both the chemical and structural contrasts. A broad range of brain tissue types has been investigated, including normal brain, different grades of gliomas, meningioma, oligodendroglioma and metastasis, with various diagnostic features of brain tumors. Machine-learning-based classifiers have been developed to provide more rapid and accurate diagnosis of these subtypes of brain tumors, pushing SRS further toward a practical tool for clinical translations.

\section{Acknowledgments}

We acknowledge the financial supports from the National Key Research and Development Program of China (2016YFC0102100, 2016YFA0301000, 2016YFA0203900); National Natural Science Foundation of China (81671725); Shanghai Rising Star Program (15QA1400500); Shanghai Action Plan for Scientific and Technological Innovation Program (16441909200).

\section{References}

1. G. Husmann, P. Kaatsch, A. Katalinic, J. Bertz, J. Haberland et al., "Krebs in Deutschland 2005/ 2006," Robert Koch-Institut und die Gesellschaft der epidemiologischen Kreberegister in Deutschland e.V., (2010).

2. P. Kremer, F. Mahmoudreza, R. Ding, M. Pritsch, S. Zoubaa et al., "Intraoperative fluorescence staining of malignant brain tumors using 5-aminofluorescein-labeled albumin," Neurosurgery 64, S53-S61 (2009).

3. K. Petrecca, M. C. Guiot, V. Panet-Raymond, L. Souhami "Failure pattern following complete resection plus radiotherapy and temozolomide is at the resection margin in patients with glioblastoma," J. Neurooncol. 111, 19-23 (2013).

4. N. Sanai, M. Y. Polley, M. W. McDermott, A. T. Parsa and M. S. Berger, "An extent of resection threshold for newly diagnosed glioblastomas," $J$. Neurosurg. 115, 3-8 (2011).

5. W. Stummer, J. C. Tonn, H. M. Mehdorn, U. Nestler, K. Franz et al., "Counterbalancing risks and gains from extended resections in malignant glioma surgery: A supplemental analysis from the randomized 5-aminolevulinic acid glioma resection study Clinical article," J. Neurosurg. 114, 613-623 (2011).

6. I. F. Talos, K. H. Zou, L. Ohno-Machado, J. G. Bhagwat, R. Kikinis et al., "Supratentorial low-grade glioma resectability: Statistical predictive analysis based on anatomic MR features and tumor characteristics," Radiology 239, 506-513 (2006).

7. L. Eisenhardt and H. Cushing, "Diagnosis of intracranial tumors by supravital technique," Am. J. Pathol. 6, 541-U531 (1930).

8. D. D. Langleben and G. M. Segall, "PET in differentiation of recurrent brain tumor from radiation injury," J. Nucl. Med. 41, 1861-1867 (2000).

9. M. C. Preul, R. Leblanc, Z. Caramanos, R. Kasrai, S. Narayanan et al., "Magnetic resonance spectroscopy guided brain tumor resection: Differentiation between recurrent glioma and radiation change in two diagnostically difficult cases," Can. J. Neurol. Sci. 25, 13-22 (1998).

10. W. Chen, "Clinical applications of PET in brain tumors," J. Nucl. Med. 48, 1468-1481 (2007).

11. Y. L. Ge, M. Law and R. I. Grossman, Applications of diffusion tensor MR imaging in multiple sclerosis, White Matter in Cognitive Neuroscience: Advances in Diffusion Tensor Imaging and Its Applications J. L. Ulmer, L. Parsons, M. Moseley, J. Gabrieli, Eds., p. 202, New York, Acad Sciences, New York (2005).

12. M. H. T. Reinges, H. H. Nguyen, T. Krings, B. O. Hutter, V. Rohde et al., "Course of brain shift during microsurgical resection of supratentorial cerebral lesions: Limits of conventional neuronavigation," Acta Neurochir. 146, 369-377 (2004).

13. M. Makary, E. A. Chiocca, N. Erminy, M. Antor, S. D. Bergese et al., "Clinical and economic outcomes of low-field intraoperative MRI-guided tumor resection neurosurgery," J. Mag. Reson. Imaging 34, 1022-1030 (2011).

14. C. Ewelt, F. W. Floeth, J. Felsberg, H. J. Steiger, M. Sabel et al., "Finding the anaplastic focus in diffuse gliomas: The value of Gd-DTPA MRI. enhanced, FET-PET, and intraoperative, ALAderived tissue fluorescence," Clin. Neurol. Neurosurg. 113, 541-547 (2011).

15. J. Regelsberger, F. Lohmann, K. Helmke and M. Westphal, "Ultrasound-guided surgery of deep seated brain lesions," Eur. J. Ultrasound 12, 115-121 (2000).

16. H. Bohringer, E. Lankenau, F. Stellmacher, E. Reusche, G. Huttmann et al., "Imaging of human brain tumor tissue by near-infrared laser coherence 
tomography," Acta Neurochir. 151, 507-517 (2009).

17. N. Sanai, J. Eschbacher, G. Hattendorf, S. W. Coons, M. C. Preul et al., "Intraoperative confocal microscopy for brain tumors: A feasibility analysis in humans," Neurosurgery 68, 282-290 (2011).

18. W. Stummer, J. C. Tonn, C. Goetz, W. Ullrich, H. Stepp et al., "5-aminolevulinic acid-derived tumor fluorescence: The diagnostic accuracy of visible fluorescence qualities as corroborated by spectrometry and histology and postoperative imaging," Neurosurgery 74, 310-319 (2014).

19. D. A. Dombeck, K. A. Kasischke, H. D. Vishwasrao, M. Ingelsson, B. T. Hyman et al., "Uniform polarity microtubule assemblies imaged in native brain tissue by second-harmonic generation microscopy," Proc. Natl. Acad. Sci. USA 100, 7081-7086 (2003).

20. S. Witte, A. Negrean, J. C. Lodder, C. P. J. de Kock, G. Testa Silva et al., "Label-free live brain imaging and targeted patching with third-harmonic generation microscopy," Proc. Natl. Acad. Sci. USA 108, 5970-5975 (2011).

21. Z. Movasaghi, S. Rehman and I. U. Rehman, "Raman spectroscopy of biological tissues," Appl. Spectrosc. Rev. 42, 493-541 (2007).

22. R. Bhargava, "Infrared spectroscopic imaging: The next generation," Appl. Spectrosc. 66, 10911120 (2012).

23. T. Meyer, N. Bergner, C. Bielecki, C. Krafft, D. Akimov et al., "Nonlinear microscopy, infrared, and Raman microspectroscopy for brain tumor analysis," J. Biomed. Opt. 16, 021113 (2011).

24. C. Krafft, L. Shapoval, S. B. Sobottka, G. Schackert and R. Salzer, "Identification of primary tumors of brain metastases by infrared spectroscopic imaging and linear discriminant analysis," Technol. Cancer Res. Treat. 5, 291-298 (2006).

25. C. Krafft, S. B. Sobottka, K. D. Geiger, G. Schackert and R. Salzer, "Classification of malignant gliomas by infrared spectroscopic imaging and linear discriminant analysis," Anal. Bioanal. Chem. 387, 1669-1677 (2007).

26. T. M. Greve, K. B. Andersen and O. F. Nielsen, "ATR-FTIR, FT-NIR and near-FT-Raman spectroscopic studies of molecular composition in human skin in vivo and pig ear skin in vitro," Spectroscopy 22, 437-457 (2008).

27. S. N. Kalkanis, R. E. Kast, M. L. Rosenblum, T. Mikkelsen, S. M. Yurgelevic et al., "Raman spectroscopy to distinguish grey matter, necrosis, and glioblastoma multiforme in frozen tissue sections," J. Neurooncol. 116, 477-485 (2014).

28. A. Mizuno, H. Kitajima, K. Kawauchi, S. Muraishi and Y. Ozaki, "Near-infrared Fourier-transform Raman-spectroscopic study of human brain-tissues and tumors," J. Raman Spectrosc. 25, 25-29 (1994).

29. M. Jermyn, K. Mok, J. Mercier, J. Desroches, J. Pichette et al., "Intraoperative brain cancer detection with Raman spectroscopy in humans," Sci. Transl. Med. 7, 274ra19 (2015).

30. H. Karabeber, R. Huang, P. Iacono, J. M. Samii, K. Pitter et al., "Guiding brain tumor resection using surface-enhanced Raman scattering nanoparticles and a hand-held Raman scanner," $A C S$ Nano 8, 9755-9766 (2014).

31. C. L. Evans, E. O. Potma, M. Puoris'haag, D. Cote, C. P. Lin et al., "Chemical imaging of tissue in vivo with video-rate coherent anti-Stokes Raman scattering microscopy," Proc. Natl. Acad. Sci. USA 102, 16807-16812 (2005).

32. B. G. Saar, C. W. Freudiger, J. Reichman, C. M. Stanley, G. R. Holtom et al., "Video-rate molecular imaging in vivo with stimulated Raman scattering," Science 330, 1368-1370 (2010).

33. A. Zumbusch, G. R. Holtom and X. S. Xie, "Threedimensional vibrational imaging by coherent anti-Stokes Raman scattering," Phys. Rev. Lett. 82, 4142-4145 (1999).

34. J. X. Cheng, A. Volkmer and X. S. Xie, "Theoretical and experimental characterization of coherent antiStokes Raman scattering microscopy," J. Opt. Soc. Am. B 19, 1363-1375 (2002).

35. J. X. Cheng and X. S. Xie, "Coherent anti-Stokes Raman scattering microscopy: Instrumentation, theory, and applications," J. Phys. Chem. B 108, 827-840 (2004).

36. E. J. Woodbury and W. K. Ng, "Ruby laser operation in the near IR," Proc. Inst. Radio Eng. 50, 2367 (1962).

37. A. Owyoung and E. D. Jones, "Stimulated Raman spectroscopy using low-power cw lasers," Opt. Lett. 1, 152-154 (1977).

38. E. Ploetz, S. Laimgruber, S. Berner, W. Zinth and P. Gilch, "Femtosecond stimulated Raman microscopy," Appl. Phys. B 87, 389-393 (2007).

39. C. W. Freudiger, W. Min, B. G. Saar, S. Lu, G. R. Holtom et al., "Label-free biomedical imaging with high sensitivity by stimulated Raman scattering microscopy," Science 322, 1857-1861 (2008).

40. D. Fu, F. K. Lu, X. Zhang, C. Freudiger, D. R. Pernik et al., "Quantitative chemical imaging with multiplex stimulated Raman scattering microscopy," J. Am. Chem. Soc. 134, 3623-3626 (2012).

41. D. A. Orringer, B. Pandian, Y. S. Niknafs, T. C. Hollon, J. Boyle et al., "Rapid intraoperative histology of unprocessed surgical specimens via fibre-laser-based stimulated Raman scattering microscopy," Nat. Biomed. Eng. 1, 0027 (2017). 
42. M. B. Ji, D. A. Orringer, C. W. Freudiger, S. Ramkissoon, X. H. Liu et al., "Rapid, label-free detection of brain tumors with stimulated Raman scattering microscopy," Sci. Transl. Med. 5, 201ra119 (2013).

43. M. B. Ji, S. Lewis, S. Camelo-Piragua, S. H. Ramkissoon, M. Snuderl et al., "Detection of human brain tumor infiltration with quantitative stimulated Raman scattering microscopy," Sci. Transl. Med. 7, 309ra163 (2015).

44. C. W. Freudiger, R. Pfannl, D. A. Orringer, B. G. Saar, M. B. Ji et al., "Multicolored stain-free histopathology with coherent Raman imaging," Lab. Invest. 92, 1661-1661 (2012).

45. F. K. Lu, S. Basu, V. Igras, M. P. Hoang, M. Ji et al., "Label-free DNA imaging in vivo with stimulated Raman scattering microscopy," Proc. Natl. Acad. Sci. USA 112, 11624-11629 (2015).

46. D. Fu, G. Holtom, C. Freudiger, X. Zhang and X. S. Xie, "Hyperspectral imaging with stimulated Raman scattering by chirped femtosecond lasers," J. Phys. Chem. B 117, 4634-4640 (2013).

47. R. He, Z. Liu, Y. Xu, W. Huang, H. Ma et al., "Stimulated Raman scattering microscopy and spectroscopy with a rapid scanning optical delay line," Opt. Lett. 42, 659-662 (2017).

48. A. Francis, K. Berry, Y. Chen, B. Figueroa and D. Fu, "Label-free pathology by spectrally sliced femtosecond stimulated Raman scattering (SRS) microscopy," PLoS One 12, e0178750 (2017).

49. F.-K. Lu, M. Ji, D. Fu, X. Ni, C. W. Freudiger et al., "Multicolor stimulated Raman scattering microscopy," Mol. Phys. 110, 1927-1932 (2012).
50. C. S. Liao, M. N. Slipchenko, P. Wang, J. Li, S. Y. Lee et al., "Microsecond scale vibrational spectroscopic imaging by multiplex stimulated Raman scattering microscopy," Light Sci. Appl. 4, e265 (2015).

51. D. Fu, "Quantitative chemical imaging with stimulated Raman scattering microscopy," Curr. Opin. Chem. Biol. 39, 24-31 (2017).

52. C. W. Freudiger, W. Yang, G. R. Holtom, N. Peyghambarian, X. S. Xie et al., "Stimulated Raman scattering microscopy with a robust fibre laser source," Nat. Photonics 8, 153-159 (2014).

53. L. Zhang, S. Shen, Z. Liu and M. Ji, "Label-free, quantitative imaging of MoS2-nanosheets in live cells with simultaneous stimulated Raman scattering and transient absorption microscopy," $A d v$. Biosyst. 1, 1700013-1700020 (2017).

54. Y. Xu, Q. Liu, R. He, X. Miao and M. Ji, "Imaging laser-triggered drug release from gold nanocages with transient absorption lifetime microscopy," ACS Appl. Mater. Interfaces 9, 19653-19661 (2017).

55. C. L. Evans, X. Xu, S. Kesari, X. S. Xie, S. T. C. Wong et al., "Chemically-selective imaging of brain structures with CARS microscopy," Opt. Express 15, 12076-12087 (2007).

56. F. K. Lu, D. Calligaris, O. I. Olubiyi, I. Norton, W. Yang et al., "Label-free neurosurgical pathology with stimulated Raman imaging," Cancer Res. 76, 3451-3462 (2016).

57. R. He, Y. Xu, L. Zhang, S. Ma, X. Wang et al., "Dual-phase stimulated Raman scattering microscopy for real-time two-color imaging," Optica 4, 4447 (2017). 